\title{
D-wave resonances in positronium hydride
}

\author{
Joseph DiRienzi \\ College of Notre Dame of Maryland, Baltimore, MD 21210
}

Richard J. Drachman

Goddard Space Flight Center, Greenbelt, MD 20771

\begin{abstract}
In a previous paper [Phys. Rev. A 65, 032721 (2002)] we re-examined a model describing the structure of the low-energy Ps-H resonances as being due to quasi-bound states of the positron in the perturbed Coulomb potential of the $\mathrm{H}^{-}$ion appearing in the closed, rearranged channel. In particular, we wished to understand why the lowest p-state resonance was so far away from the lowest quasi-bound $(2 p)$ state. We found that the lowest resonance actually corresponds to the first excited [3p] state, while the lowest state is not recognizable as a resonance. In the present work we repeat our analysis, but this time for the lowest $d$ state. We find that the lowest [3d] state does correspond to a resonance shifted moderately.

PACS numbers: $34.50 .-\mathrm{s}, 36.10 . \mathrm{Dr}$
\end{abstract}


There has been much recent theoretical interest in the positronium-hydrogen [Ps- $\mathrm{H}]$ scattering system [1], and special interest has focused on the properties of elastic scattering resnnances that have been predicted by a variety of theoretical techniques $[2,3]$. In particular, the model of resonances due to hydrogenic bound states in the re-arranged $\left[\mathrm{e}^{+}-\mathrm{H}^{-}\right]$ channel predicts the existence of an infinite sequence of resonances in all angular-momentum states [4]. We may expect these resonances to lie fairly close to the levels of hydrogen although perturbed by the non-Coulomb parts of the negative hydrogen ion's potential. In addition, the L-degeneracy of the hydrogen levels should be broken by this short-range repulsive potential so as to raise the low-L states more than the higher ones, since they are more penetrating. A simple S-wave calculation based on this idea was carried out long ago [4], including the coupling between the hydrogenic bound states and the scattering continuum. The lowest S-state was not expected to correspond to a resonance, since there is a single $\mathrm{S}$-wave bound state in Ps-H, but the $2 s$ state did generate a resonance that agreed well with other calculations [2]. Based on this success, it was assumed that the correspondence between hydrogenic bound states and resonances would carry over trivially to higher values of the angular momentum.

Therefore it was a surprise when the lowest $P$ and $D$ resonances were found [3] to be radically shifted from their expected positions, based on the hydrogenic model. That was our motivation for re-examining that model, including P-states in our analysis. We were again surprised to find [5] that the $2 p$ state was broadened out of all recognition, and that it was actually the $3 p$ state that produced the lowest $\mathrm{P}$ resonance, at a position in good agreement with the results of Ref.[3]. Since we now know that the lowest state in both S- and $\mathrm{P}$-waves is ineffective at producing scattering resonances, we wish to find out whether this situation is true for higher- $\mathrm{L}$ waves too. In this Brief Report we outline the corresponding calculation for $L=2$. In this case, we find that the lowest state does in fact correspond to the lowest resonance, in contrast to the lower- $\mathrm{L}$ cases.

The method described in Ref. $[4,5]$ is essentially a two-state approximation:

$$
\Psi\left(\vec{x}, \vec{r}_{1}, \vec{r}_{2}\right)=F\left(\vec{R}_{1}\right) \phi\left(\rho_{1}\right) \psi\left(r_{2}\right)+\{1 \leftrightarrow 2\}+G(\vec{x}) \Phi\left(r_{1}, r_{2}\right)
$$

where

$$
G(\vec{x})=\frac{g(x)}{x} P_{2}(\hat{x} \cdot \hat{z})
$$


and the ground states of Ps and $\mathrm{H}$ atoms have the following forms:

$$
\phi(\rho)=\frac{e^{-\rho / 2}}{\sqrt{8 \pi}}, \psi(r)=\frac{e^{-\tau}}{\sqrt{\pi}} .
$$

The function $F(\vec{R})$ represents the Ps-H scattering, both atoms being unexcited, while $G(\vec{x})$ is the wave function of the positron bound in a d-state to the negative hydrogen ion. The system is in the singlet electronic spin state (corresponding to the symmetry of the ionic bound state $\left.\Phi\left(r_{1}, r_{2}\right)\right)$ and is explicitly antisymmetrized.

In Ref.[5] the details of the variational derivation of an integro-differential equation for $F(\vec{R})$ are described. This has the following form:

$$
-\frac{1}{2}\left[\nabla^{2}+k^{2}\right] F(\vec{R})+\int d \vec{R}^{\prime} K\left(\vec{R}, \vec{R}^{\prime}\right) F\left(\vec{R}^{\prime}\right)+2 \sum_{N} \frac{V_{N d}(\vec{R}) \int d \vec{R}^{\prime} V_{N d}\left(\vec{R}^{\prime}\right) F\left(\vec{R}^{\prime}\right)}{E-E_{H^{-}}-\epsilon_{N d}}=0 .
$$

Two major simplifications are then made: First, the integral kernel coming from the exchange of the two electrons is replaced by an effective separable form

$$
K\left(\vec{R}, \vec{R}^{\prime}\right)=b e^{-\alpha\left(R+R^{\prime}\right)} R R^{\prime} P_{2}(\hat{R} \cdot \hat{z}) P_{2}\left(\hat{R}^{\prime} \cdot \hat{z}\right)
$$

and second, the hydrogen ion wave function is replaced by its simplest approximation

$$
\Phi\left(\hat{r}_{1}, \hat{r}_{2}\right)=\frac{Z^{3}}{\pi} e^{-Z\left(r_{1}+r_{2}\right)}, Z=\frac{11}{16} .
$$

The first step is to omit the closed-channel function $G(\vec{x})$ which removes the optical potential terms from Eq.(4) and solve the resulting static-exchange equation:

$$
-u^{\prime \prime}(R)-k^{2} u(R)+\frac{6 u(R)}{r^{2}}+\lambda e^{-\alpha R} R^{2} \int_{0}^{\infty} d R^{\prime} R^{\prime 2} e^{-\alpha R^{\prime}} u\left(R^{\prime}\right)=0,
$$

where $\lambda=8 \pi b / 5$ and $F(\vec{R})=u(R) P_{2}(\hat{R} \cdot \hat{z}) / R$. This equation has the following solution:

$$
u(R)=\vec{j}(k R)-\tan \eta\left[\bar{n}(k R)+e^{-\alpha R} \sum_{n=0}^{4} C_{n} R^{n-2}\right],
$$

where

$$
C_{0}=\frac{3}{k^{2}}, C_{1}=\frac{3 \alpha}{k^{2}}, C_{2}=\frac{3 \alpha^{2}+k^{2}}{2 k^{2}}, C_{3}=\frac{\alpha\left(\alpha^{2}+k^{2}\right)}{2 k^{2}}, C_{4}=\frac{\left(\alpha^{2}+k^{2}\right)^{2}}{8 k^{2}}
$$

and

$$
\begin{aligned}
& \bar{j}(z)=\frac{3 \sin z}{z^{2}}-\frac{3 \cos z}{z}-\sin z \\
& \bar{n}(z)=-\frac{3 \cos z}{z^{2}}-\frac{3 \sin z}{z}+\cos z .
\end{aligned}
$$


The effective range formula takes the form

$$
k^{5} \cot \eta=-\frac{\left(\alpha^{2}+k^{2}\right)^{6}}{64 \lambda}+\frac{\left(k^{2}-\alpha^{2}\right)}{256 \alpha^{5}}\left[3 \alpha^{8}+28 \alpha^{6} k^{2}+178 \alpha^{4} k^{4}+28 \alpha^{2} k^{6}+3 k^{8}\right] .
$$

By fitting this expression to the $L=2$ phase shifts calculated in the static-exchange approximation Ref.[6] we find a best fit for $\alpha=1.30244$ and $\lambda=-1.02029$, and we will assume that the separable form of the exchange kernel shown in Eq.(5) is appropriate even when we add the correlated terms coming from the closed-channel hydrogen-ion parts of the wave function of Eq.(1).

The virtual bound state wave function $G(\vec{x})$ can consist of one or more D-state eigenfunctions of the differential equation

$$
-g_{N d}^{\prime \prime}(x)+\frac{6}{x^{2}} g_{N d}(x)+U(x) g_{N d}(x)=\epsilon_{N d} g_{N d}(x),
$$

where $U(x)$ is the potential due to the simple form of $\Phi$ shown in Eq.(6):

$$
U(x)=-\frac{2}{x}+4 e^{-2 Z x}\left(\frac{1}{x}+Z\right) .
$$

The two lowest eigenvalues are $\epsilon_{3 d}=-0.1097 R y$ and $\epsilon_{4 d}=-0.0618 R y$, which lie very close to the unperturbed hydrogenic values of $-1 / 9$ and $-1 / 16$. We are interested in finding how close to these positions the corresponding scattering resonances lie. (Note that the second of these lies above the inelastic threshold of the Ps atom at $\epsilon=-3 / 8-E_{H^{-}}+E_{H}+E_{P_{s}}=$ -0.0695 .)

We follow the same procedure in calculating the separable potentials $V_{N d}(\vec{R})$ as for the earlier S- and P-wave calculations of Ref.[5]:

$$
V_{N d}\left(\vec{R}_{1}\right)=\iint d \vec{\rho}_{1} d \vec{r}_{2} \phi\left(\rho_{1}\right) \psi\left(r_{2}\right) \frac{g_{N d}(x)}{x} P_{2}(\hat{x} \cdot \tilde{z}) \Phi\left(r_{1}, r_{2}\right)\left[\Delta_{N d}+\frac{2}{x}-U(x)-\frac{2}{\rho_{1}}-\frac{2}{\rho_{2}}\right] .
$$

Here $\Delta_{N d}=E_{H^{-}}+\epsilon_{N d}-E_{H}-E_{P s}-k^{2} / 2$, so that for the lowest resonance $\Delta_{3 d}=0.3348-$ $k^{2} / 2$. Once the potentials have been evaluated the numerical solution of the scattering equation by the noniterative method is quite straightforward.

The potential $V_{N d}\left(\vec{R}_{1}\right)$ is the result of an integration over the two remaining independent Jacobi coordinates describing the system, $\vec{\rho}_{1}$ and $\vec{r}_{2}$. But there are additional coordinates involved in the integration, especially the argument of the Legendre polynomial giving the angular dependence of the bound state function $G(\vec{x})$. We expect the angular dependence 
of the optical potential to involve only D-states of the variable $\mu_{R}=\hat{R}_{1} \cdot \hat{z}$, so the variables in the integrand must be appropriately transformed. (The integration over $d \vec{r}_{2}$ is trivial.)

Since $\vec{x}=\vec{R}_{1}+\vec{\rho}_{1} / 2$ we can write

$$
\hat{x} \cdot \hat{z}=\frac{1}{x}\left(R_{1} \mu_{R}+\frac{1}{2} \rho_{1} \mu_{\rho}\right)
$$

where we define $\mu_{\rho}=\hat{\rho}_{1} \cdot \hat{z}$ and $\mu=\hat{\rho}_{1} \cdot \hat{R}_{1}$. The first term in Eq.(14) has the desired form, and we can write the second in terms of

$$
\mu_{\rho}=\frac{4 \pi}{3} \sum_{m=-1}^{I} Y_{1}^{m}\left(\mu_{R}, \phi_{R}\right) Y_{1}^{-m}(\mu, \phi)=\mu_{R} \mu-\sqrt{\left(1-\mu_{R}^{2}\right)\left(1-\mu^{2}\right)} \cos \left(\phi_{R}-\phi\right)
$$

In our previous work on P-states, at this point we could neglect the $\phi$-dependent terms which would vanish after integration over $d \phi$. But in the present case we must construct $P_{2}(\hat{x} \cdot \hat{z})$ which is quadratic in its argument, and the quadratic $\phi$-dependent terms do not vanish after integration. After some algebra we obtain the transformed expression

$$
P_{2}(\hat{x} \cdot \hat{z})=\frac{P_{2}\left(\mu_{R}\right)}{x^{2}}\left[R_{1}^{2}+R_{1} \rho_{1} \mu+\frac{\rho_{1}^{2}}{4} P_{2}(\mu)\right] .
$$

Finally, we can now write the potential explicitly in terms of a double integral:

$$
V_{N d}(\vec{R})=4 \sqrt{2}\left(\frac{Z}{Z+1}\right)^{3} P_{2}\left(\mu_{R}\right) \int_{0}^{\infty} d \rho \rho^{2} e^{-\frac{\rho}{2}} \int_{-1}^{1} d \mu e^{-Z r} \frac{g(x)}{x^{3}} Q\left(\Delta_{N d}+P\right) .
$$

Here we have used the definitions

$$
Q=R^{2}+R \rho \nu+\frac{1}{4} \rho^{2} P_{2}(\mu)
$$

which comes obviously from Eq.(16), and

$$
P=e^{-(1+Z) x}\left(\frac{2}{x}+1+Z\right)-U(x)-\frac{2}{\rho} .
$$

Everything has now been re-written in terms of the three variables $R, \rho$, and $\mu$, keeping in mind that $[x, r]=\sqrt{R^{2}+\rho^{2} / 4 \pm R \rho \mu}$. In practice we separate $V_{N d}(\vec{R})$ into two parts, one independent of energy and one proportional to $\Delta_{N d}$, carry out the double integrals numerically for a range of values of $R$, and then fit the results smoothly by interpolation. The resulting potentials are then used in the scattering equation (4), after expansion in partial waves, to compute the $D$-wave phase shift as a function of energy.

The results are not as surprising as were those presented in Ref.[5]. In Fig.1 we show the phase shift when only the $3 d$ closed-channel state is included in $G(\vec{x})$. In this case a single, 


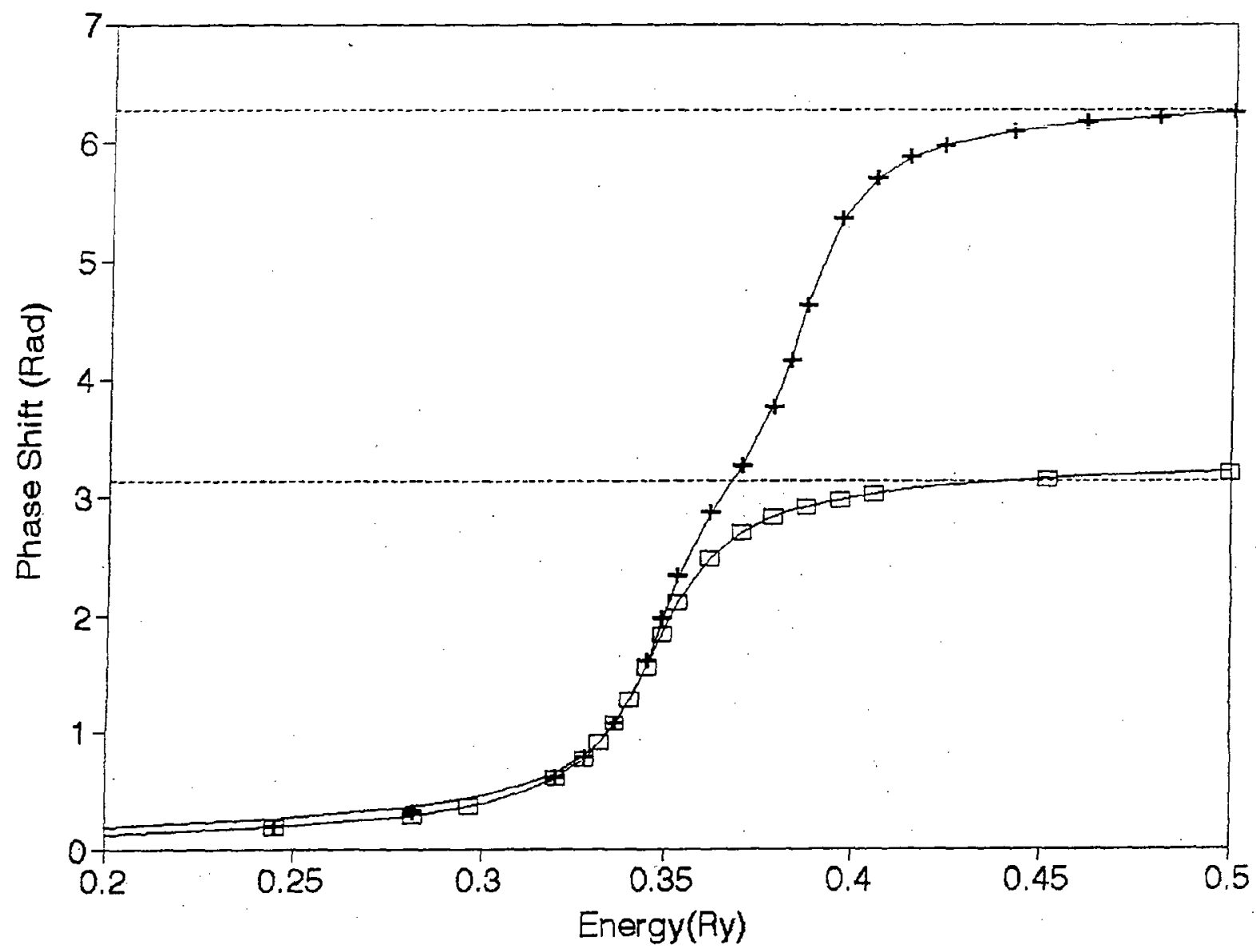

FIG. 1: Ps-H D-wave phase shifts. The squares are the result when only the $3 \mathrm{~d}$ state is included, and the crosses are the result when the $4 \mathrm{~d}$ state is added. The curves are best-fit analytical functions of the standard form [Eq.(18)]. The two horizontal lines are at $\pi$ and $2 \pi$, respectively, to guide the eye.

fairly narrow resonant feature is seen, and when it is fitted with the standard resonance formula

$$
\eta(E)=A+B E+\arctan \left[\frac{\Gamma}{2\left(E_{R}-E\right)}\right]
$$

we find the results shown in Table I. (The energies there are the Ps-H scattering energies $k^{2} / 2$, not energies measured relative to $\mathrm{H}^{-}$). You can see that the resonance energy obtained this way is very close to the result in [4], although the width is too large.

Also in Fig.1 we plot the phase shift when we add the $4 d$ state. Here we find two resonances, which lie quite close together. The results of a two-term fit are also shown in Table I, although the higher one is perhaps not too significant since it lies above the Ps 
TABLE I: D-wave resonances in various approximations. The energies $\epsilon_{N d}$ do not include coupling to the conimum. The lowest resonance is labeled 1 and the second is 2.

\begin{tabular}{|c|c|c|}
\hline Method & Ps scattering energy $(\mathrm{eV})$ & Width (eV) \\
\hline$\epsilon_{3 d}$ & 4.555 & \\
$\epsilon_{4 d}$ & 5.207 & \\
$\mathrm{E}_{1}(1$ term $)$ & 4.708 & 0.394 \\
$\mathrm{E}_{1}(2$ term) & 4.729 & 0.327 \\
$\mathrm{E}_{1}[4]$ & $4.710 \pm 0.0027$ & $0.0925 \pm 0.0054$ \\
$\mathrm{E}_{2}(2$ term) & 5.253 & 0.266 \\
\hline \hline
\end{tabular}

inelastic threshold of $0.375 \mathrm{Ry}$ and has not been previously calculated. With the inclusion of this second state the position of the first resonance rises slightly and is in somewhat poorer agreement with Ref.[4], while its narrower width is a slight improvement.

We conclude from these calculations that the $\mathrm{D}$-wave resonances behave almost exactly as we would have expected before the problem with the P-wave scattering arose. That is, coupling with the continuum shifts the position of the lowest resonance, but only by a modest amount. There is no sign of the phenomenon that startled us in the P-wave work, where the lowest potential resonance disappeared due to that coupling. We now feel confident that calculations of higher- $L$ resonances (which we do not intend to carry out) will continue to act normally, although their positions above the inelastic threshold would necessitate multi-channel formulations.

[1] C. P. Campbell, M. T. McAlinden, F. G. R. S. MacDonald, and H. R. J. Walters, Phys. Rev. Lett. 80, 5097 (1998); I. A. Ivanov, J. Mitroy, and K. Varga, ibid, 87, 063201 (2001); D. Bressanini, M. Mella, and G. Morosi, Phys. Rev. A 57, 1678 (1998); S. K. Adhikari and P. Mandal, J. Phys. B 34, L187 (2001).

[2] R. J. Drachman and S. K. Houston, Phys. Rev. A 12, 885 (1975); Y. K. Ho, ibid. 17, 1675 (1978).

[3] Z.-C. Yan and Y. K. Ho, Phys. Rev. A 57, R2270 (1998); Y. K. Ho and Z.-C. Yan, J. Phys. B 31, L877 (1998). 
[4] R. J. Drachman, Phys. Rev. A 19, 1900 (1979).

[5] J. DiRienzi and R. J. Drachman, Phys. Rev. A 65, 032721 (2002).

[6] S. Hara and P. A. Fraser; J. Phys. B 8, L472 (1975); H. Ray and A. S. Ghosh, J. Phys. B 29, $5505(1996) ; 30,3745(1997)$. 